\title{
Modeling and simulation of ion energy distribution functions in technological plasmas
}

\author{
Thomas Mussenbrock \\ Institute of Theoretical Electrical Engineering, Ruhr University Bochum, D-44780 Bochum, Germany
}

Key words Capacitive Discharge, Radio-frequency Plasma, Plasma Sheath, Ion Energy Distribution Function

The highly advanced treatment of surfaces as etching and deposition is mainly enabled by the extraordinary properties of technological plasmas. The primary factors that influence these processes are the flux and the energy of various species, particularly ions, that impinge the substrate surface. These features can be theoretically described using the ion energy distribution function (IEDF). The article is intended to summarize the fundamental concepts of modeling and simulation of IEDFs from simplified models to self-consistent plasma simulations. Finally, concepts for controlling the IEDF are discussed.

(c) 2008 WILEY-VCH Verlag GmbH \& Co. KGaA, Weinheim

\section{Introduction}

It was almost ten years before the advent of plasma physics in the beginning of the 1930th by Langmuir and Tonks that it was claimed that energetic ions should play an important role for the deposition of metals. In 1920, The Physical Society of London and The Optical Society discussed "The Making of Reflecting Surfaces". It was argued that "the production of mirrors by cathodic bombardment is not a new process". Since Sir William Grove, who is better known as one of the two fathers of the fuel cell, discovered the phenomenon of sputtering in 1852, workers with vacuum tubes have noticed the bright deposit in the vicinity of electrodes. It was found that this is more or less due to the current passed through the tube and the physical nature of the gas. They were convinced that the mechanism of the process is doubtless that particles of the metal are projected from the cathode by electric repulsion of like charges. However, the discharges itself were crude apparatus and the actual mechanism of sputtering was not understood.

Within the next fifty years - until the 1960th - the basic concepts of today's low-temperature low-pressure plasma sources and processes were developed. One important milestone was the introduction of parallel plate capacitively coupled radio-frequency discharges (CCPs) which were then widely used to generate energetic ions for sputter etching and also sputter deposition. Results from the first measurements of ion energy distribution functions (IEDFs) were published by Erö in 1958. He performed these measurements in the context of research on a radio-frequency driven ion source of Thoneman type, which is an inductively coupled plasma source [1]. A typical IEDF from the measurements of Erö is depicted in figure 1. It shows the typical bimodal shape due to the radio-frequency modulation with a breadth of $55 \mathrm{eV}$. Another very important milestone which strongly influenced later research activities on IEDFs has been published in 1963. Butler and Kino studied the plasma sheath formation by radio-frequency fields [2]. In this context the role of the external circuit, i.e., the blocking capacitor and the formation of a DC self-bias voltage were discussed for the first time.

In the late 1960th and the early 1970th CCPs were mainly applied for plasma etching or sputtering. Plasma etching was more or less isotropic and combined volatile products and low ion energies. On the other hand sputtering combined nonvolatile products and high ion energies. It was actually the time that the need for volatile etch product and anisotropic etch (high ion energies) was recognized. In 1974 Hosokawa et al. were the first who combined volatile products and high ion energies driven by their interest to realize faster plasma etching [3]. They found that a fluorine plasma of a CCP can be a very useful source of both energetic ion fluxes and reactive neutral species when the substrate is placed on the powered electrode. It is of course due to the sheath which is formed between the substrate and the plasma and in which the highly mobile electrons are absent to form a strong electric field allowing an energetic flux of positively charged particles to the wall. The quite sharp potential 


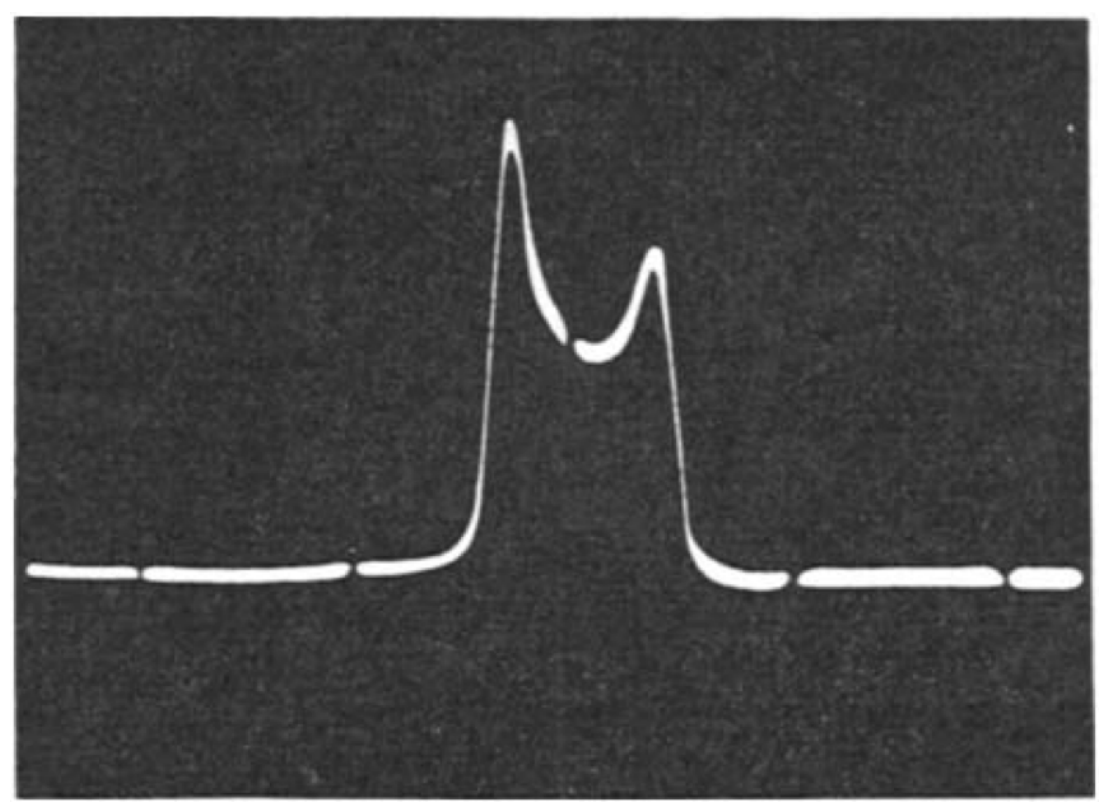

Fig. 1 Energy spectrum at $3 \mathrm{keV}$ from Erö [1]. The figure shows the characteristic bimodal shape due to the radio-frequency modulation. The distance between the two maxima corresponds to $55 \mathrm{eV}$.

gradient causes the positive ion flux to maintain a narrow angular distribution at the wafer which ultimately leads to anistropic etching.

At least since Coburn and Winters in 1979 published their famous work on plasma-assisted surface treatment, low-temperature low-pressure plasmas began to revolutionize the materials processing scene [4]. Coburn and Winters found that a synergistic effect appears when combining an argon ion beam with a fluorine atom flux from a hot $\mathrm{XeF}_{2}$ gas. They showed in their famous figure (figure 2) that due to the combined fluxes the etch rate is an order of magnitude larger than that produced by either individual flux. It was also found by Coburn and Winters that the required activation of the process by the ion flux allowed the anisotropic nature of the etching process to be retained. This actually could be named the advent of reactive ion etching (RIE), or even the advent of plasma-based materials processing. Driven by the quest of the semiconductor industry to further satisfy Moore's law, plasma processing plays nowadays a crucial role for the fabrication of most of the available hi-tech products.

In order to allow for anisotropic etching at high etch rate, modern plasma sources are operated at high plasma densities and low gas pressures. The sheath voltages are often quite small to prevent ion induced damaging of the structured substrates. In this regime the mean width of the sheath is much smaller than the mean free path of the ions. Therefore the ions traverse the sheath without any collision and the spread of related IEDFs and IADFs (ion angular distribution functions) is minimal. At higher pressures the number of collisions of ions and neutrals of the background become significant. The result is a very complex and broad shape of the IEDF in contrast to the beam-like distribution at low pressures.

Over the last decades a large number of modeling and simulation approaches to low-temperature low-pressure plasmas, to their sheaths, and inherently to IEDFs have been published. (An excellent review has been povided by Kawamura et al. in 1999 [5].) Actually, it is impossible to explicitly acknowledge all activities in this field. That is why this manuscript makes no claim to be complete. In contrast, this paper is intended to summarize the fundamental concepts of modeling and simulation of IEDFs from simplified models to self-consistent plasma simulations. It is therefore organized as follows: After this introductory part, the basic simplified models for calculating IEDFs are revisited. It will be argued that the dynamics of sheath play a crucial role for the characteristics of the IEDF. Therefore, the Brinkmann sheath model is briefly discussed. The following fourth chapter deals with selfconsistent plasma simulations in the context IEDF calculations. Finally, well-established and new concepts for controlling the IEDF are discussed. 


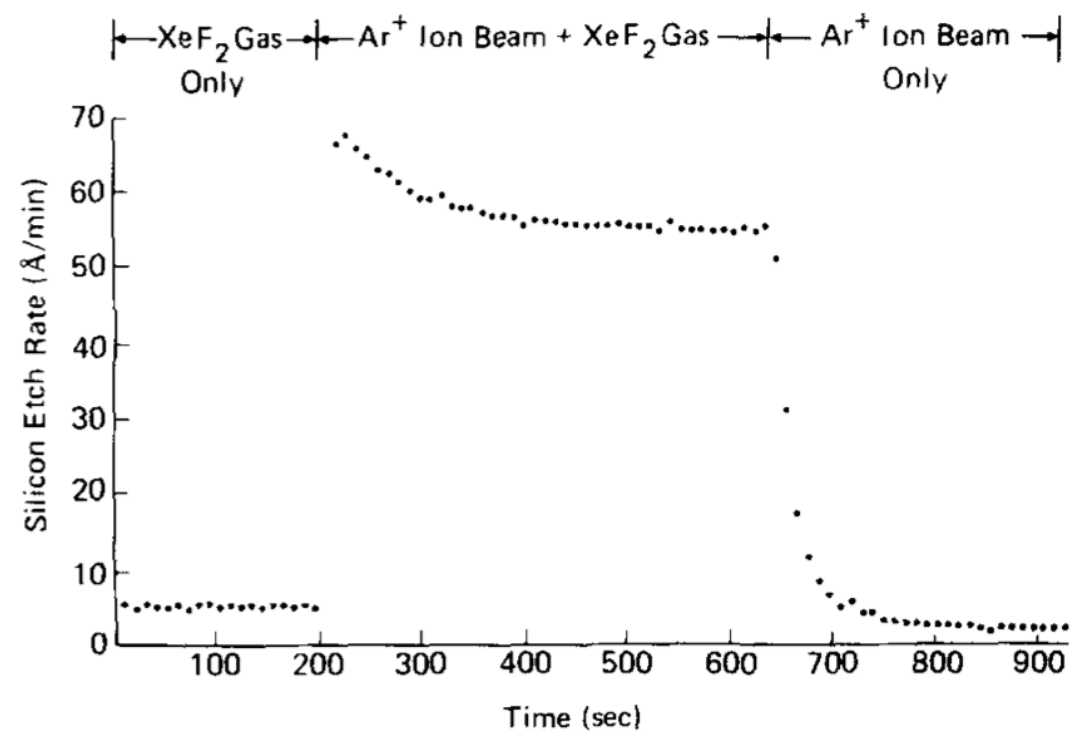

Fig. 2 Ion-assisted etching of a silicon substrate using either argon ions or xenon difluorid gas, or both at the same time from Coburn and Winters [4].

\section{Simplified models}

Analytical models for calculating IEDFs are often based on the simplifying assumption of a collisionless sheath. The mean free path of the ions is assumed to be much smaller than the mean sheath width. In this context important parameters are the ratio between the ion transit time $\tau_{i o n}$ (the time for an ion traverse through the sheath) and the time constant of the frequency of the sheath modulation $\tau_{r f}$, which is nearly the time constant of the driving radio-frequency. Assuming a Child-Langmuir sheath with a sheath potential $V_{s} \propto x^{4 / 3}$, one obtains

$$
\frac{\tau_{i o n}}{\tau_{r f}}=\frac{3 \bar{s} \omega_{r f}}{2 \pi} \sqrt{\frac{m_{i}}{2 e \bar{V}_{s}}},
$$

with $\bar{s}, \bar{V}_{s}$, and $m_{i}$ being the mean sheath width, the mean sheath voltage, and the ion mass, respectively.

One may distinguish between two regimes: The high-frequency regime where $\tau_{i o n} / \tau_{r f} \gg 1$ and the lowfrequency regime where $\tau_{i o n} / \tau_{r f} \ll 1$. In the latter regime the ions traverse the sheath within a very short time, which can be only a very small fraction of one low-frequency period. Therefore the ions experience the instantaneous electric field of the sheath. The IEDF at the electrode is strongly depending on the phase of the electric field at the instant time when the ions arrive at the sheath edge. In the low-frequency regime the IEDF has more or less the bimodal shape, similar to the IEDF which is displayed in figure 1. The bimodal shape is characterized by the distance between the two maxima $\Delta E$. The maxima of the IEDF correspond with the minimum and the maximum of the sheath voltage, respectively. In the high-frequency regime the ions traverse through the sheath within in a large number of radio-frequency cycles. The ions experience more or less the timeaveraged electric field rather than the instantaneous electric field. In this regime one obtains a narrow, beam-like IEDF. It has been shown that for the high-frequency regime the breadth of the IEDF is directly proportional to the ratio $\tau_{i o n} / \tau_{r f}$. If $\tau_{i o n}$ increases for fixed $\tau_{r f}, \Delta E$ becomes smaller. For a very large ion transit time the bimodal IEDF becomes a monoenergetic IEDF.

The first analytical calculation of the IEDF at a substrate was proposed by Benoit-Catin and Bernard for the collisionless high-frequency regime [6]. The model is based on a number of simplifying assumptions: In the frame of a Child-Langmuir sheath model the sheath width is assumed to be constant and the electric field is assumed to be uniform. The temporal modulation of the sheath voltage is harmonic, i.e., $V_{s}=\bar{V}_{s}+\hat{V}_{s} \sin \omega_{r f} t$. Additionally, it is assumed that the ions start with zero initial velocity at the sheath edge. A straight forward analysis of Poisson's equation coupled with Newton's law of motion for a charged particle in an electric field 


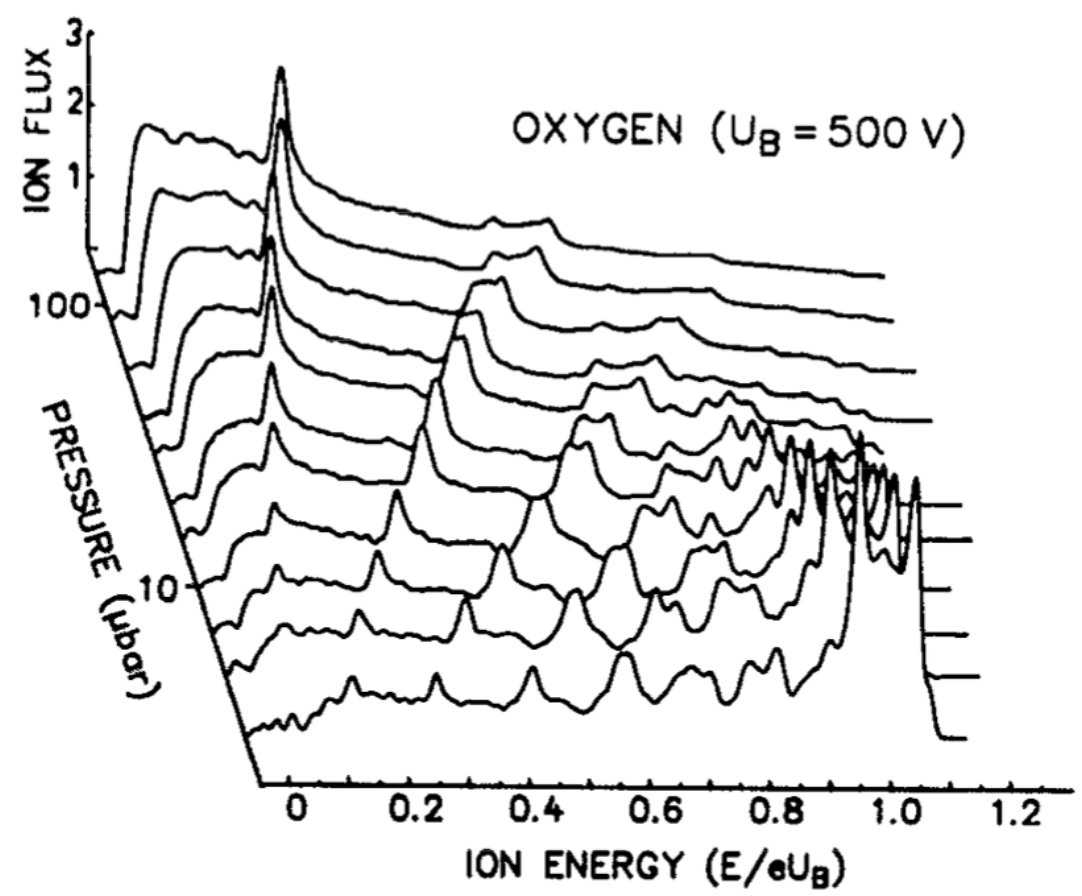

Fig. 3 Ion energy distributions measured in oxygen discharges at different pressures from Wild and Koidl 7

yields an expression for the IEDF $f(E)$ and the energy spread $\Delta E$, respectively,

$$
f(E)=\frac{2 n_{t} / \omega_{r f} \Delta E}{\sqrt{1-\frac{4}{\Delta E^{2}}\left(E-e \bar{V}_{s}\right)^{2}}}
$$

and

$$
\Delta E=\frac{8 e \tilde{V}_{s}}{3 \bar{s} \omega_{r f}} \sqrt{\frac{2 e \bar{V}_{s}}{M}} .
$$

$f(E)$ shows the typical bimodal shape with two maxima symmetrically localized about $e \bar{V}_{s}$. The energy spread $\Delta E$ is proportional to $\tau_{r f} / \tau_{i o n}$. With increasing frequency or increasing ion mass, $\Delta E$ becomes smaller. Ultimately, the two peaks approach to each other and become one monoenergetic peak. Due to the monoenergetic initial velocity distribution one obtains singular behavior of $f(E)$ at

$$
E=e \bar{V}_{s} \pm \frac{\Delta E}{2}
$$

The effect of collisions of ions with the neutrals of the background gas within the sheath was first studied experimentally as well as theoretically by Wild and Koidl [7]. Figure 3 shows a series of measured IEDFs in oxygen discharges at different gas pressures. One can observe a number of peaks and double peaks whose occurrence strongly depend on the pressure. At low pressures the IEDF shows mainly the well-known bimodal structure at high energies and a number of less significant peaks for lower energies. This structure is due to ions which have traversed the sheath without collisions. With increasing pressures, the bimodal shape successively disappears and the average ion energy decreases significantly.

The theoretical model proposed by Wild and Koidl is able to resolve for the complex multi-peak structure of the IEDFs. It is based on similar simplifying assumptions as the collisionless model of Benoit-Catin and Bernard. All assumptions are well justified and can be applied to the collisional case. Of course, the most important issue in this context is the consideration of collisions of ions with neutrals during their traverse of the sheath. Of all 


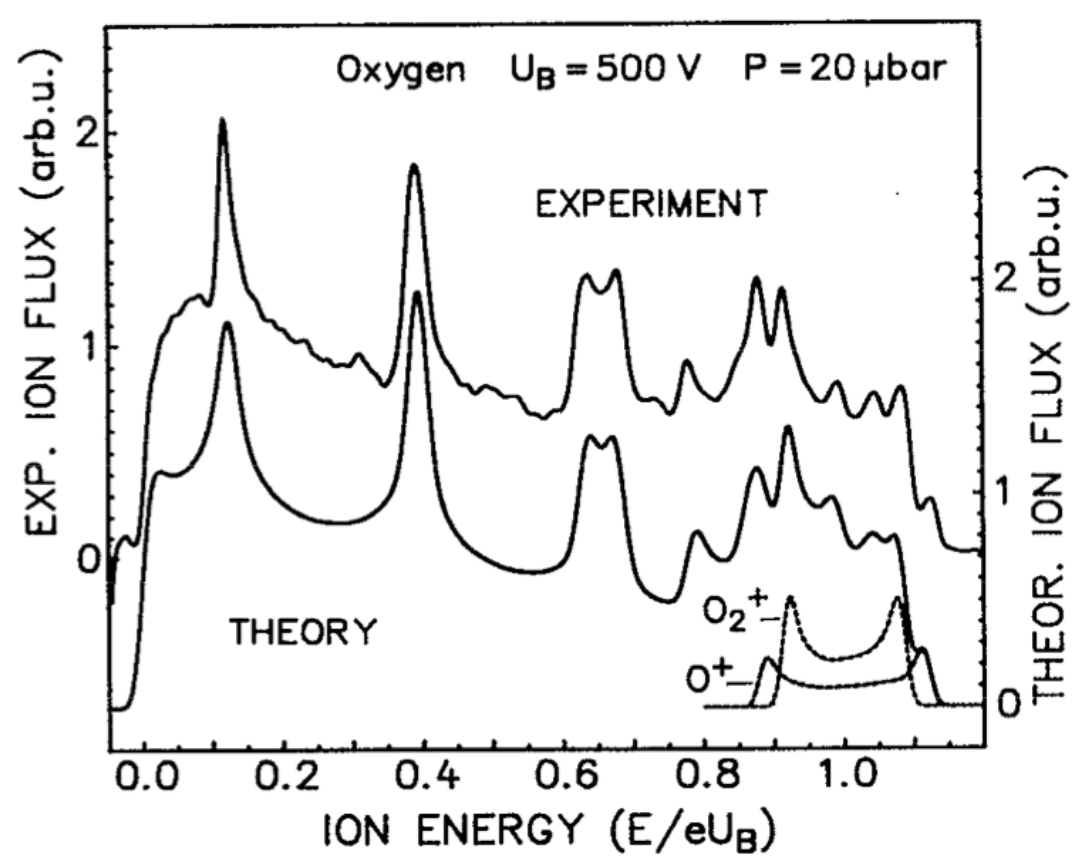

Fig. 4 Experimental (upper curve) and theoretical (lower curve) energy distribution of the ions in an oxygen discharge from Wild and Koidl [7]. The small dashed lines in the lower right corner represent the contribution of the unscattered ions to the theoretical IEDF.

ion collision processes within the sheath, the charge exchange collision between the ions and the neutrals have the largest cross-section. Thus, charge exchange processes play the major role and are exclusively allowed for. It can be found that even at low pressures the charge exchange process is quite probable and dominant. It should not be neglected when studying the ion transport through the sheath.

The charge exchange collision of an energetic ion with a thermalized neutral particle produces an energetic neutral and a thermalized ion under conservation of the total ion flux. The assumption is that a thermalized ion is generated at the position $p_{0}$. The probability that this particular ion reaches the substrate is given by $\exp \left[\alpha\left(1-p_{0}\right)\right]$ with $\alpha$ being the ratio between the mean sheath width and the ion mean free path within the sheath. The energy of the ions that impinge on the substrate is therefore a function of the position of the collision $p_{0}$ and the instantaneous phase of the electric field $\phi_{0}$ at the instant of time of the collision. If ions traverse the sheath without any collision, the energy is a function of the initial velocity $v_{0}$ at the sheath edge and the instantaneous phase $\phi_{0}$ of the electric field. For a constant collision cross section one arrives at

$$
f(E) d E \propto \int \mathrm{e}^{-\alpha\left(1-p_{0}\right)} d p_{0} d \phi_{0}+\mathrm{e}^{-\alpha} \int f\left(v_{0}\right) d v_{0} d \phi_{0}
$$

which is an expression for the IEDF allowing for charge exchange collisions of ions within the sheath. Here $f\left(v_{0}\right)$ is the distribution of the initial velocity of ions at the sheath edge. The first part of this expression represents the contribution of ions which are produced due to a charge exchange process within the sheath. The second term is the contribution of ions which traverse the sheath without any collision. It can be shown that the initial ion velocity distribution at the sheath edge has almost no influence on the IEDF at the substrate. Therefore $f\left(v_{0}\right)$ is often assumed to be a Boltzmann distribution. Figure 4 shows both experimental and theoretical results for IEDFs in an oxygen discharge. Whereas the upper curve shows the experimental result, the lower curve is the result from the model. The small dashed lines in the lower right corner represent the contribution of the unscattered ions to the theoretical IEDF. One can find that the results are in almost perfect agreement. In the displayed case of an oxygen discharge one can observe a shoulder in the experimental results for high energies which can not be resolved when assuming only singly ionized oxygen molecules $\mathrm{O}_{2}^{+}$. To account for this structure, the contribution of ionized oxygen atoms $\mathrm{O}^{+}$or double ionized oxygen molecules $\mathrm{O}_{2}^{2+}$ have to be included into the model. 


\section{Self-constistent sheath models}

As already mentioned in the previous chapters the most important parameter - besides the gas mixture and gas pressure - is the electric field within the sheath region. To calculate the spatial and temporal dynamics of the sheath (and inherently the electric field) a large number of stand-alone sheath models have been proposed, e.g., by Lieberman, Biehler, Riemann, or Godyak [8-13]. All models are more or less able to resolve for the important phenomena. A very elegant and powerful model has been developed by Brinkmann [14-16]. The model is able to treat an arbitrary number of positive and negative ions and is therefore appropriate in the context of technological applications. Brinkmann's model is characterized by a smooth transition from unipolarity (in the sheath) to ambipolarity (in the bulk plasma), instead of a step-like transition. Capacitively driven sheaths exhibit a smooth transition, which is expanded by the radio-frequency modulation and smoothed by thermal effects which are namely the finiteness of the electron temperature and the Debye length. Sheath models which neglect thermal effects are characterized by a step in the spatial electron density distribution. These so-called step models are restricted to strongly modulated high voltage sheaths with $V_{R F} \gg T_{e} / e$ and fail when this condition is not met. To overcome this problem, Brinkmann proposed an improved model of the sheath-bulk transition, which takes into account both modulation and thermal effects. Based on an asymptotic solution of the Boltzmann-Poisson equation one can derive an approximate algebraic expressions for the phase-resolved electrical field and electron density in radio-frequency driven sheaths. Under the assumption that the modulation of the sheath is periodic with $\omega_{R F} \gg \omega_{p i}$, also the phase averages of the electric field and the electron density can be explicitly expressed in closed form. These results - together referred to as the Advanced Algebraic Approximation (AAA) - make it possible to formulate efficient and accurate models for radio frequency driven boundary sheaths for all ratios $e V_{R F} / T e$ and for an arbitrary number of positive and negative ions.

Kratzer et al. applied the AAA to self-consistently calculate the electric field for a number of different plasma parameters and gas mixtures [17]. The electric field has been then used in a Monte Carlo module in order to calculate the ion trajectories within the sheath. The collisions of the ions with the neutrals of the background gas are treated in the frame of the null collision method. The related IEDFs from Brinkmann's sheath model have been impressively validated. Figure 5 shows both numerical and experimental results for IEDFs in a radiofrequency oxygen discharge at a gas pressure of 3 mTorr. The experimental results were originally taken from Kuypers and Hopman [18]. To compare the experimental data with the simulation results, the latter have been smoothed with a Gaussian smoothing filter of $3 \mathrm{eV}$ FWHM which was roughly the average resolution in the experimental data. In both results one can identify two distinct double peak structures which are due to the two positive oxygen ion species $\mathrm{O}_{2}^{+}$and $\mathrm{O}^{+}$. Whereas the inner peaks belong to $\mathrm{O}_{2}^{+}$, the species with the higher mass, the outer lying peaks belong to the lighter ions $\mathrm{O}^{+}$. In accordance with the theory described above the peak separation is inversely proportional to the square root of the ion masses

$$
\frac{\Delta E_{1}}{\Delta E_{2}} \propto \sqrt{\frac{m_{2}}{m_{1}}}
$$

The calculated IEDF by means of Brinkmann's AAA displayed in figure 5 reproduce the experimental measurements in great detail. Of course, the assumption that the ion plasma frequency is much larger than modulation frequency of the sheath is a limitation of the AAA in its current stage. However, the remedy of this drawback is a topic of ongoing research.

\section{Self-consistent discharge models}

Although stand-alone sheath models are able to provide meaningful results for a wide range of plasma parameters, all these models have an intrinsic drawback: They are not fully self-consistent in the sense that the ion flux and energy at the sheath edge has to be assumed. This drawback can be remedied by using fully self-consistent discharge models. A large number of such models, which are based on different approaches, are available. The main difference is manifested in the fact, whether the approach is fluid dynamic, kinetic, or hybrid (which means both fluid and kinetic).

A very prominent and elaborated hybrid model is the Hybrid Plasma Equipment Model (HPEM) which has been implemented over the last 30 years by Kushner and co-workers [19]. It is devoted to provide a simulation 

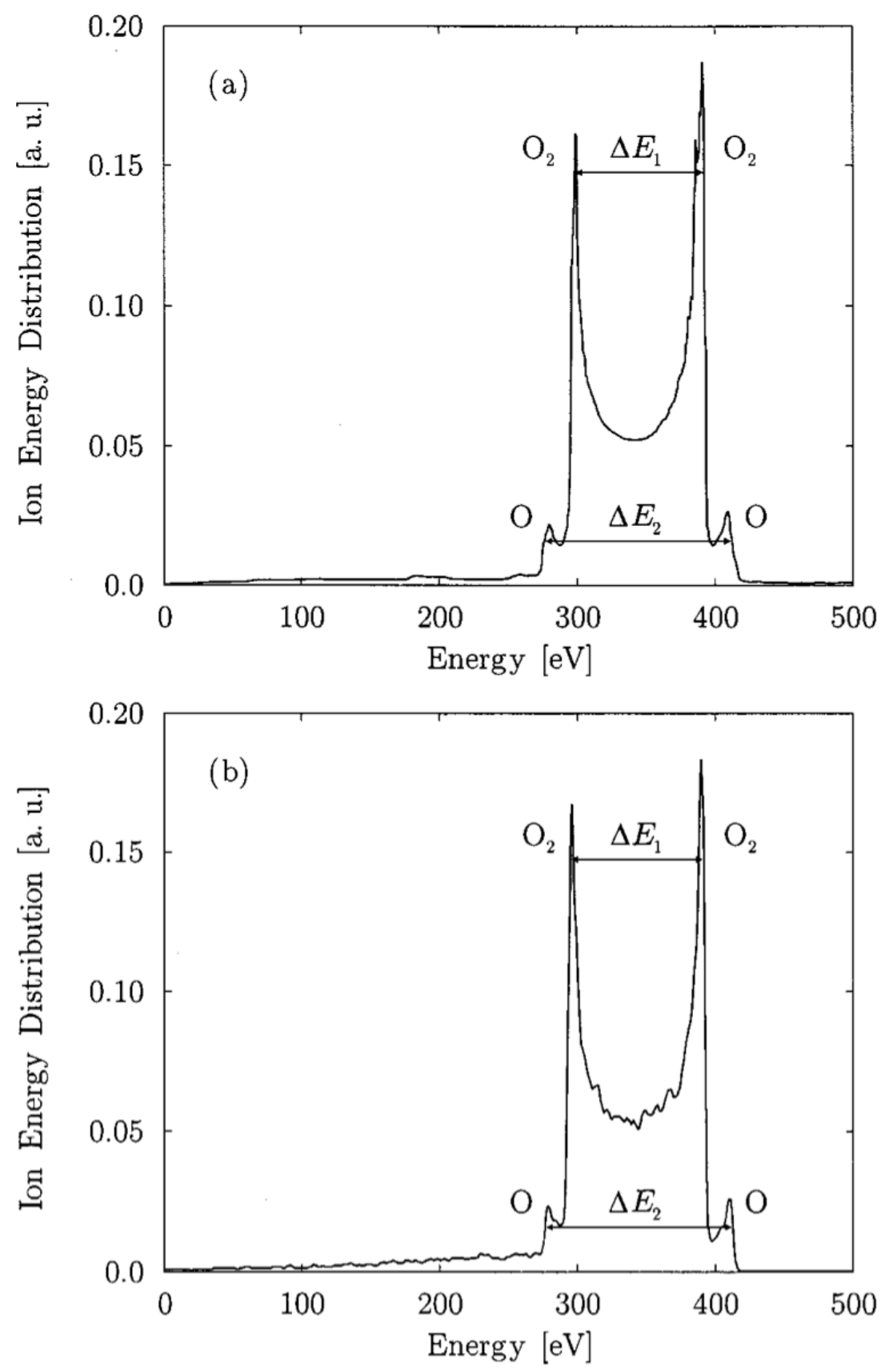

Fig. 5 Calculated and measured IEDFs of an oxygen discharge at 3 mTorr from Kratzer et al. [17]. The experimental result obtained by Kuypers and Hopman is displayed above [18]. The corresponding simulation result is given below.

tool for low-temperature low-pressure plasma systems, from external circuits to the feature length scale in order to simulate plasma based surface processes. A huge number of researcher and applicants from the industry have successfully applied HPEM, not only to understand the basic plasma physics but also to optimize industrial plasma processes. Similar to other available hybrid simulation models, HPEM consists of a number of distinct modules. Basically, it self-consistently solves continuity, momentum, and energy equations for neutrals and ions, continuity equations for electrons, and Maxwell's equations. It utilizes an electron Monte Carlo simulation to obtain the electron energy distribution functions of bulk electrons and secondary electrons emitted from surfaces. 

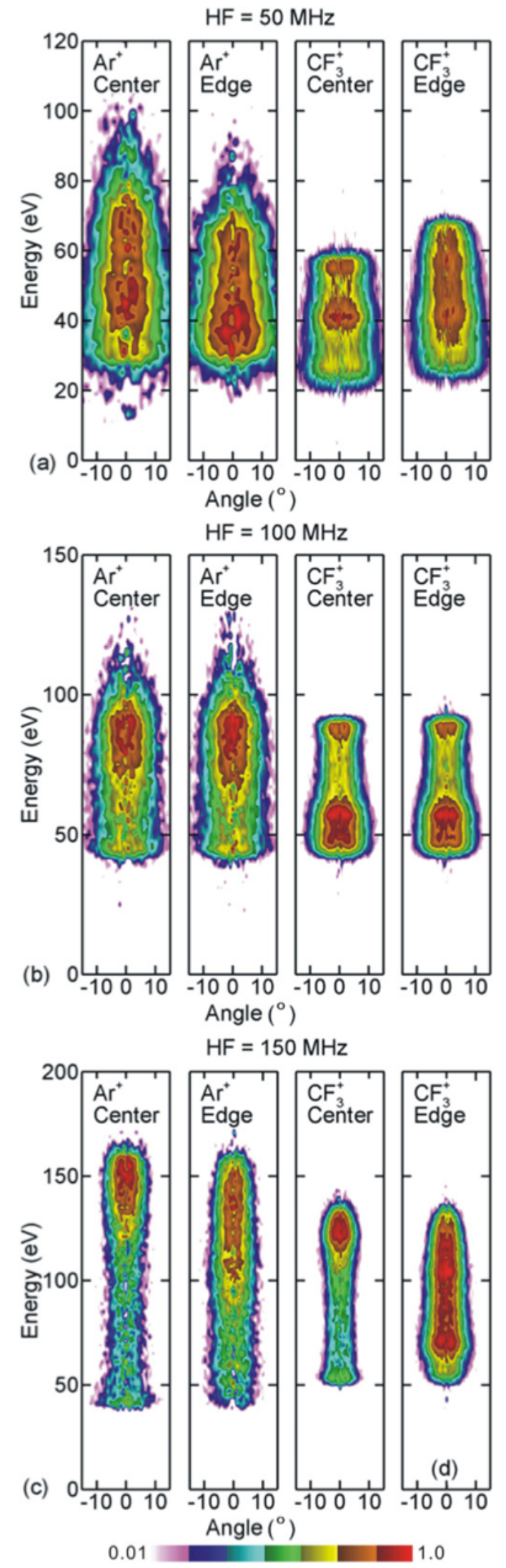

(c) 2008 WILEY-VCH Verlag GmbH \& Co. KGaA, Weinheim
Fig. 6 IEDFs of different ion species in an $\mathrm{Ar} / \mathrm{CF}_{4}$ plasma obtained by using HPEM for different dual-frequency excitation from Yang and Kushner [21] 


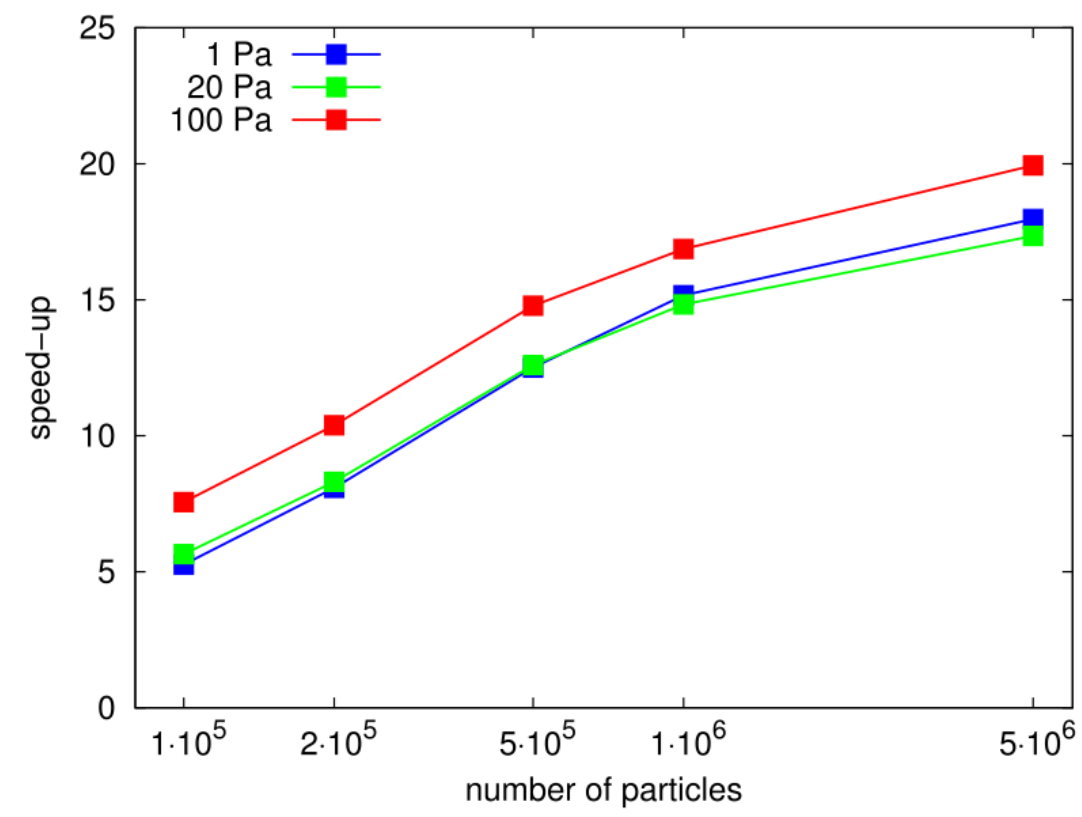

Fig. 7 Speed-up of the fine-sorted PIC algorithm, compared to a CPU (single core) approach as a function of the number of superparticles for different pressures from Mertmann et al. [29].

The equations are integrated in time over many radio-frequency cycles to obtain a periodic steady state. During the last iteration, the converged electric fields and source functions for ions and neutral are recorded as a function of position and phase in the radio-frequency cycle. With these values, the energy and angular distributions of ions and neutrals incident on the substrate are obtained using an adapted plasma chemistry module based on a Monte Carlo scheme [20]. The IEDFs can then be used to perform simulations of the actual plasma processes on the length scale of the feature, e.g., the etching or deposition of materials.

Figure 6 shows typical HPEM results for IEDFs of different ion species in an $\mathrm{Ar} / \mathrm{CF}_{4}$ plasma [21]. The IEDFs of $\mathrm{Ar}$ and $\mathrm{CF}_{3}$ ions as functions of the angle of impact on the substrate are displayed for a dual frequency excitation of $10 \mathrm{MHz} / 50 \mathrm{MHz}, 10 \mathrm{MHz} / 100 \mathrm{MHz}$, and $10 \mathrm{MHz} / 150 \mathrm{MHz}$ (see next section). One can observe an increasing radial dependence of the maximum of the ion energy as the high-frequency increases. This phenomenon can be explained by non-uniform plasma distributions at 50 and $150 \mathrm{MHz}$ due to electromagnetic effects as the standing wave effect and the skin effect $[22-25]$.

However, although hybrid models provide very convincing results, the most straightforward way to simulate plasmas is the particle-in-cell (PIC) method pioneered among others by Birdsall [26] and firstly applied to low-temperature plasmas by Vender and Boswell [27]. They set up a PIC model allowing for charge exchange collisions in the frame of the Monte Carlo (MC) scheme of a 1D parallel plate radio-frequency discharge in hydrogen. It was intended to study the IEDF at the electrode for a number ofdifferent parameters. The results confirmed both, the analytical results for the simplified collisionless model proposed by Benoit-Catin and the numerical results performed by Koidl and Wild using the collisional model. It was Surendra and Graves who first implemented a collision model included elastic and ionizing electron neutral collisions in addition to charge exchange collisions of ions [28]. They conducted simulations of radio-frequency discharges in Helium which also confirmed the experimental results of Koidl and Wild.

Since the basic concept of the particle in cell method coupled to Monte Carlo collisions is quite simple, a large number of researchers and research groups implemented their own PIC code in order to have their own numerical "plasma experiment". The advantage of the PIC concept is that the fields and the energy distribution can be obtained from first principles. There is no need for simplifying assumptions for the fields or the distributions. However, the advantage comes along with a significant disadvantage. The PIC/MC scheme tends to be computationally very expensive compared to other numerical simulation methods. The good news is that modern computers have become very fast and very large memories are affordable. Particularly, modern graphics cards 

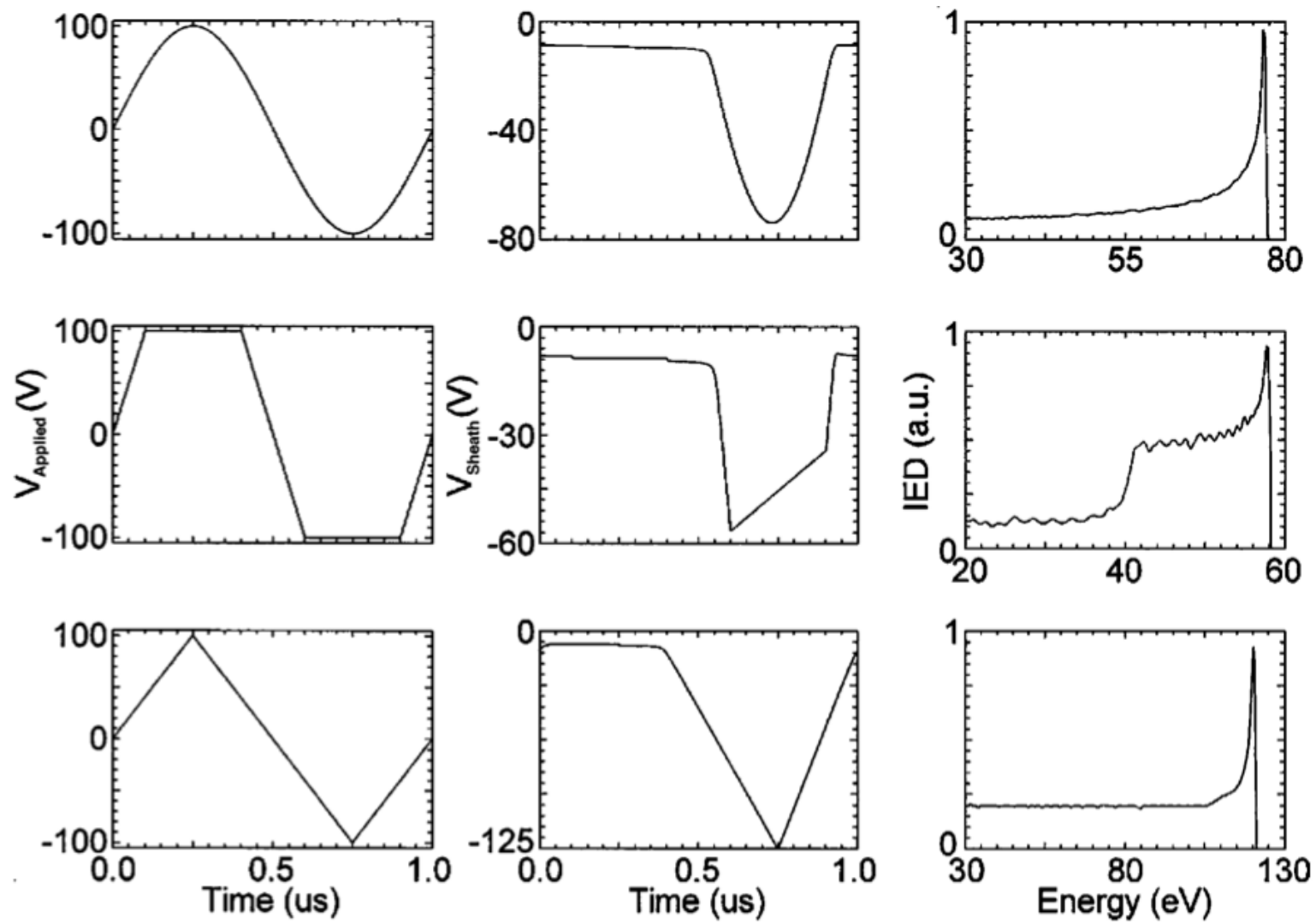

Fig. 8 Applied radio-frequency bias voltage (left column), sheath voltage (center collumn), and IEDF (right column) for sinusoidal (above row), square (center row), and triangular waveforms (below row) from Rauf [30]. The results has been obtained for inductively coupled plasma in Argon at $20 \mathrm{mTorr}$ with substrate bias voltage of $100 \mathrm{~V}$ at $1 \mathrm{MHz}$.

(or graphics processing units GPUs), originally introduced to make computer games more realistic and more fast, provide a very strong computational infrastructure. Up to 1.000 cores on one GPU are available. It has been shown that PIC/MC codes in running parallel on standard GPUs can easily reach a speed-up of nearly a factor of 80 compared to the serial PIC code for CPUs. A second inherent advantage is that a very large number of particles (up to a few 10 millions) can be simply tracked without losing significant performance.

However, despite all technical advances which have been recently made in the context of PIC/MC, there is still lack of knowledge about collision cross section which are needed to implement reasonable collision processes in the frame of the Monte Carlo scheme. If one is interested in more complex gas mixtures, e.g., which are used for plasma surface treatment, one would probably use fluid models or hybrid models.

\section{Control of IEDFs}

The ability of modeling and simulation the IEDF in technological plasmas forces the quest to actually control the energy and the flux of the ions impinging on the substrate in order to directly control plasma surface interactions. A number of methods which are intended to control (or at least to adjust) the features of the IEDF have been developed, or are still matter of ongoing research.

As mentioned in the previous chapters, it is the sheath voltage which mainly determines the IEDF. The sheath voltage itself can be adjusted using a bias voltage at the substrate. In this context Rauf explored the influence of the waveform and frequency of a radio-frequency bias voltage on the IEDF [30]. Using the HPEM, Rauf found - as expected - that the waveform adjusts the shape of the IEDF while the frequency determines its breadth. He performed numerical experiments by varying the waveform of the bias voltage. In figure 8 the results for three 


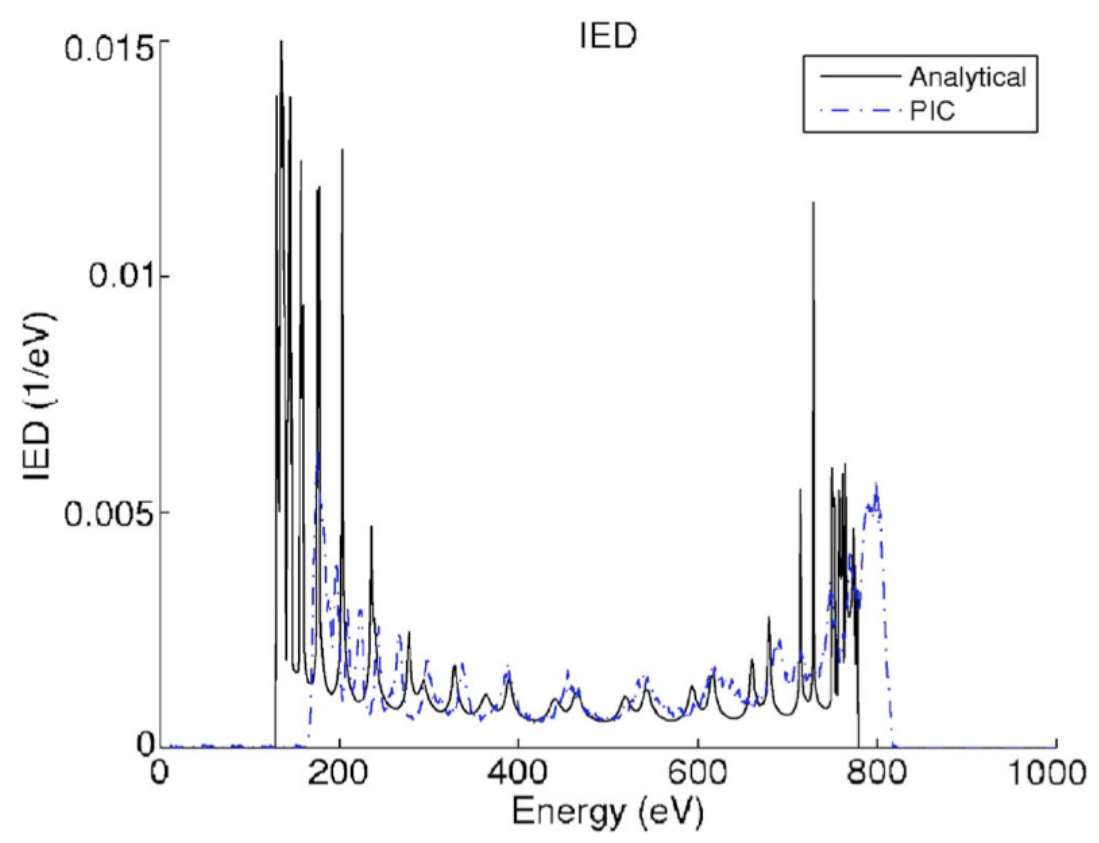

Fig. 9 IEDF calculated by the analytical model compared to results from a PIC/MC simulation for an argon discharge driven by two radio-frequency voltages $(800 \mathrm{~V}$ at $2 \mathrm{MHz}$ and $400 \mathrm{~V}$ at $64 \mathrm{MHz})$ from $\mathrm{Wu}$ et al [31].

different waveforms are displayed. It can be observed that a sinusoidal waveform leads to an IEDF that peaks at high energies and gradually decreases with decreasing energy. A square waveform results in a sharp step in the IEDF at high energies. The triangular waveform generates a constant IEDF over a large range of energy.

Rauf also proposed in his paper that one can design voltage waveforms that produce IEDFs with specific features by utilizing the correlation between them. This idea has been revisited by Wu et al. [31]. They developed an analytical model which is used to predict the IEDF given the sheath voltage. They assume that the response of the ions $V_{i}(t)$ is determined by $V_{i}(f)=\alpha(f) V_{s}(f)$, with $V_{i}(f)$ and $V_{s}(f)$ being the Fourier transforms of $V_{i}(t)$ and $V_{s}(t)$, respectively. $\alpha(f)$ is the transfer function which is defined by

$$
\alpha(f)=\frac{1}{\left[\left(c f \tau_{i}\right)^{p}+1\right]^{1 / p}}
$$

The transfer function is chosen in way that the ions have a $\left(f \tau_{i}\right)^{-1}$ dependence at high frequencies and completely respond to low frequency oscillations. The constants $c$ and $p$ are fitting parameters. Once the ion response voltage $V_{i}(t)$ is calculated, it will be converted into the corresponding IEDF using

$$
f(E) \propto \sum_{j}\left|\frac{d V_{i}}{d t_{j}}\right|^{-1},
$$

with $j$ being the energy interval. Figure 9 shows an analytically calculated IEDF compared to results from a $\mathrm{PIC} / \mathrm{MC}$ simulation for an argon discharge driven by two radio-frequency voltages ( $800 \mathrm{~V}$ at $2 \mathrm{MHz}$ and $400 \mathrm{~V}$ at $64 \mathrm{MHz}$ ). The analytical IEDF is shown as the solid line, and the PIC/MC result is shown as the dashed line. One can observe that for the dual-frequency excitation of the discharge the analytical model is able to predict the IEDF, including its substructures. Calculations of IEDFs performed for other dual- and triple frequency cases show similarly good agreements.

Two (or even more) distinct radio-frequencies are not only used for biasing the substrate. Particularly two radio-frequency voltages have been successfully introduced in order to power the discharge itself. The so-called dual-frequency CCP concept helps to remedy the significant drawback of conventional single-frequency CCPs that the ion bombardment energy and the ion flux to the substrate cannot be controlled independently. Goto et 

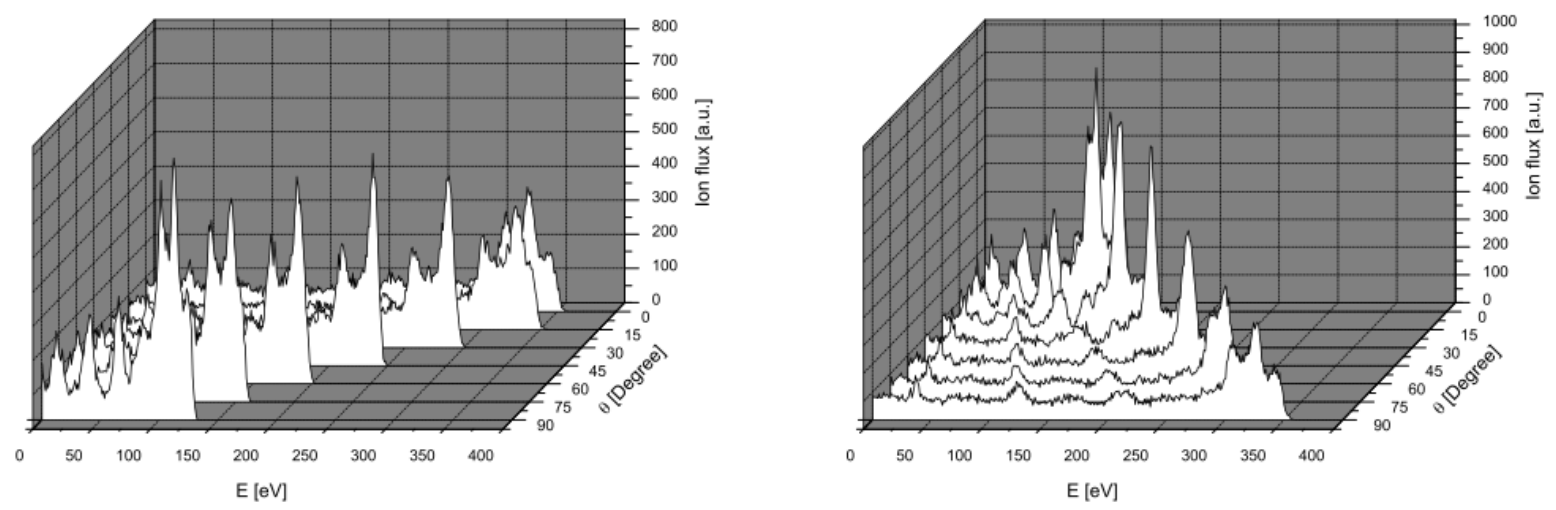

Fig. 10 Ion flux-energy distributions at the driven (left) and grounded (right) electrode as a function of the phase angle $\theta$ obtained by PIC/MCC simulations from Donko et al. [42].

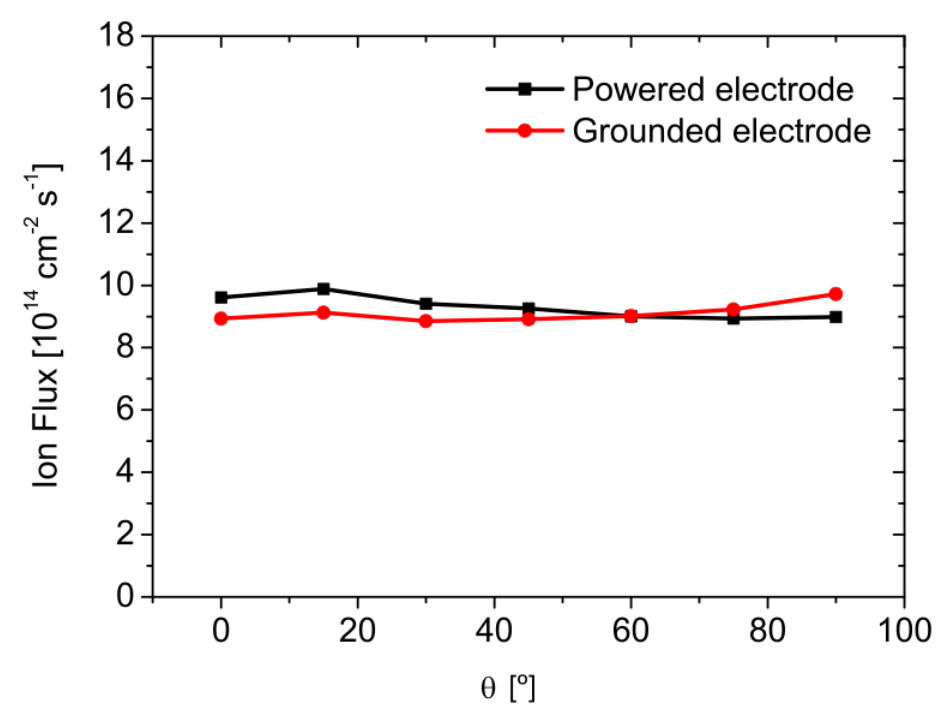

Fig. 11 Ion fluxes at the driven (left) and grounded (right) electrode as a function of the phase angle $\theta$ obtained by PIC/MCC simulations from Donko et al. [42][49].

al. [32] as well as Nakano and Makabe [33] found that under certain conditions dual-frequency CCPs allow for an almost independent control of the ion flux by the high frequency power and the ion bombardment energy by the low frequency power. In principle, the mechanism is quite simple: The source operating at the high frequency "sees" the sheath capacitances as a shortcut; its voltage drops across the bulk, where it leads to Ohmic dissipation, electron heating, and ionization. The low frequency voltage, on the other hand, drops mostly across the sheath; its peak value determines the sheath voltage and thus the ion energy. For a more detailed discussion see Lee et al. [34, 35], who conducted numerical investigations on the control of the IEDF in dual-frequency CCPs. In $2 \mathrm{f}-\mathrm{CCPs}$, the ratio of the applied frequencies $\omega_{H F} / \omega_{L F}$ is obviously an important control parameter. By means of simulations using HPEM, Rauf and Kushner found that at large ratios $\omega_{H F} / \omega_{L F} \gg 10$ the spectrum of the radio-frequency current through the discharge is just the superposition of two single frequency spectra [36]. For more comparable frequencies $\left(\omega_{H F} / \omega_{L F} \lesssim 10\right.$, however, quite surprising nonlinear effects were observed, which are indicated by the appearance of harmonics and sidebands in the spectrum [37-39].

These nonlinear effects can lead under certain conditions to a strong coupling between the two frequencies, that might limit separate control of the ion flux and energy. This drawback can be overcome by making use of the electrical asymmetry effect (EAE). With the help of the EAE an almost strictly separate control of the ion energy and flux at the electrode surfaces can be achieved in a simple way. The EAE can be found in dual-frequency 

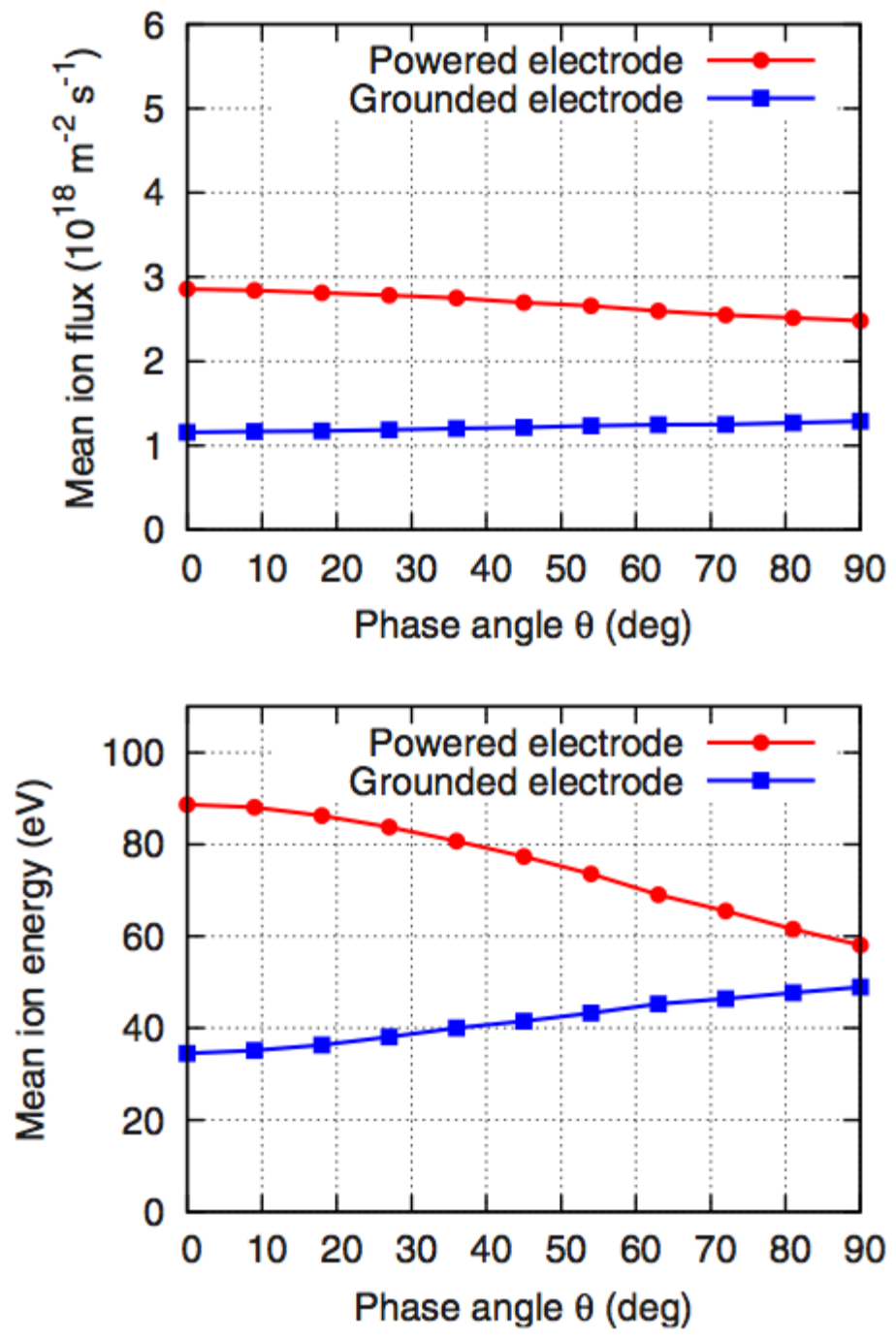

Fig. 12 Mean flux (top) and mean energy (bottom) of ions at the smaller powered electrode (red line and dots) and at the larger grounded electrode (blue line and squares) from Schulze et al. [44]

CCPs driven at comparable frequencies, e.g., 13.56 MHz and 27.12 MHz [40-43]. If the electrode is driven at a fundamental frequency and its second harmonic with variable phase shift between the two voltage waveforms, $V_{R F}=\hat{V}_{R F}[\cos (\omega t+\theta)+\cos 2 \omega t]$, one obtains a DC self bias voltage as a function of the phase angle $\theta$. This holds even for geometrically symmetric CCPs were the driven electrode and the grounded electrode (including grounded walls) are of the same size. This variable DC self bias adjusted by the phase controls the ion energy at the electrodes (or the substrate). As only the relative phase between the voltage harmonics but not their amplitude is changed, the ion flux at the electrodes remains constant.

Based on an analytical model developed by Heil et al. [40], one can formulate an expression for the DC self bias voltage $\eta$,

$$
\eta=-\frac{\phi_{\max }+\varepsilon \phi_{\min }}{1+\varepsilon}
$$


Here, $\phi_{\max }$ and $\phi_{\min }$ is the absolute maximum and absolute minimum of the applied driving voltage waveform, respectively. $\varepsilon$ is the so-called symmetry parameter defined by

$$
\varepsilon=\left|\frac{\phi_{s g}}{\phi_{s p}}\right| \approx\left(\frac{A_{p}}{A_{g}}\right)^{2} \frac{\bar{n}_{s p}}{\bar{n}_{s g}}\left(\frac{Q_{m g}}{Q_{m p}}\right)^{2} .
$$

$\phi_{s g}, \phi_{s p}$ is the maximum voltage drop across the sheath at the grounded and powered electrode, respectively, $\bar{n}_{s p}$, $\bar{n}_{s g}$ is the spatially averaged ion density, and $Q_{m g}, Q_{m p}$ is the maximum charge in the respective sheath. The remarkable feature of the EAE is that the DC self bias is an almost linear function of the phase angle between the driving frequencies in range between 0 and $\pi / 2$. This has been confirmed my PIC/MC simulation and fluid models. Therefore the phase angle $\theta$ is knob for tuning the IEDF. Donko et al performed 1D PIC/MC simulations of an geometrically symmetric argon discharge driven at $13.56 \mathrm{MHz}$ and $27.12 \mathrm{MHz}$ [42]. Figure 10 shows the obtained IEDFs in front of both the driven electrode (right) and the grounded electrode (left). One can observe the effect of varying the phase angle $\theta$ on the IEDF at both electrodes. By changing $\theta$ from 0 to $\pi / 2$ the maximum ion energy at both electrodes can be changed by a factor of almost 3. Furthermore, the role of each electrode can be reversed by changing $\theta$. The high ion energy at the driven electrode at $\theta=0$ can be switched to the grounded electrode at $\theta=\pi / 2$, and vice versa. Another important feature of this behavior is the fact that the switching from high to low ion energy (and from low to high ion energy) can be realized under almost constant ion flux. Figure 11 shows the ion flux at both electrodes as the phase angle $\theta$ is varied from 0 to $\pi / 2$. The ion flux remains constant within $\pm 5 \%$, while the maximum ion energy changes by a factor of 3 as $\theta$ changes. The observed stability of the ion flux is within the range of tolerance for most industrial applications.

The EAE can also be used to reduce the asymmetry of geometrically asymmetric discharges. By tuning the phase angle shift between two consecutive driving harmonics, the absolute value of the DC self-bias voltage can be substantially reduced and the mean ion energies at both electrodes can be made similar. The related results from PIC/MC simulations are displayed in figure 12. It could be very interesting to work out whether this effect similarly appears in discharges in molecular, electronegative gases which are used for materials processing. This might allow to switch electrically from an etching mode to a cleaning or even a deposition mode in one and the same reactor. One would avoid the necessity for a change of the gas mixture for chemical wall cleaning and different chamber geometries for etching and deposition processes.

\section{Conclusion}

The fundamental concepts of modeling and simulation of IEDFs from simplified models to self-consistent plasma simulations are summarized. Finally well-established and new concepts for controlling the IEDF are discussed. Although the overall number of contributions to the field of IEDF modeling and simulation is tremendous and research has been done for more than 40 years it is still a topic of growing interest. This holds particularly for the control of IEDFs and even the closed-loop control and tailoring IEDFs pioneered by Wendt and coworkers [45,-48] and improved by Baloniak and von Keudell [49-51] in order to realize specific properties of substrate materials. Unfortunately, this exciting topic is beyond the scope of this paper. However, the basis of all concepts is adapted modeling of IEDFs.

\section{Acknowledgement}

The author gratefully acknowledges numerous discussions with Ralf Peter Brinkmann and the people of the Institute of Theoretical Electrical Engineering at the Ruhr University Bochum. The work is supported by the Deutsche Forschungsgemeinschaft DFG in the frame of SFB-TR 87.

\section{References}

[1] J. Erö, Nucl. Instr. 3, 303 (1958)

[2] H.S. Butler and G.S. Kino, Phys. Fluids. 6, 1346 (1963)

[3] N. Hosokawa, R. Matsuzaki, and T. Asamaki, Jpn. J. Appl. Phys. Suppl. 2, 435 (1974)

[4] J.W. Coburn and H.F. Winters, J. Appl. Phys. 50, 3189 (1979) 
[5] E. Kawamura, V. Vahedi, M.A. Lieberman, and C.K. Birdsall, Plasma Sources Sci. Technol. 8, R45 (1999)

[6] P. Benoit-Catin and L.C. Bernard, J. Appl. Phys. 39, 5723 (1968)

[7] C. Wild and P. Koidl, J. Appl. Phys. 69, 2909 (1991)

[8] M.A. Lieberman, IEEE Trans. Plasma Sci. 16, 638 (1988)

[9] M.A. Lieberman, IEEE Trans. Plasma Sci. 17, 338 (1989)

[10] S. Biehler, Appl. Phys. Lett. 54, 317 (1989)

[11] K.-U. Riemann, J. Appl. Phys. 65, 999 (1989)

[12] V.A. Godyak and N. Sternberg, Phys. Rev. A 42, 2299 (1990)

[13] V.A. Godyak, R.B. Piejak, and N. Sternberg, IEEE Trans. Plasma Sci. 21, 378 (1993)

[14] R.P. Brinkmann, J. Appl. Phys. 102, 093303 (2007)

[15] R.P. Brinkmann, J. Phys. D: Appl. Phys. 42, 19400 (2009)

[16] R.P. Brinkmann, J. Phys. D: Appl. Phys. 44, 042002 (2011)

[17] M. Kratzer, R.P. Brinkmann, W. Sabisch, and H. Schmidt, J. Appl. Phys. 90, 2169 (2001)

[18] A.D. Kuypers and H.J. Hopman, J. Appl. Phys. 67, 1229 (1990)

[19] M.J. Kushner, J. Phys. D: Appl. Phys. 42, 194013 (2009)

[20] J. Lu and M.J. Kushner, J. Vac. Sci. Technol. A 19. 2652 (2001)

[21] Y. Yang and M.J. Kushner, Plasma Sources Sci. Technol. 19, 055011 (2010)

[22] M.A. Lieberman, J.P. Booth, P. Chabert, J.M. Rax, and M.M. Turner, Plasma Sources Sci. Technol. 11, 283 (2002)

[23] P. Chabert, J. Phys. D: Appl. Phys. 40, R63 (2007)

[24] T. Mussenbrock, T. Hemke, D. Ziegler, R.P. Brinkmann, and M. Klick, Plasma Sources Sci. Technol. 17, 025018 (2008)

[25] I. Lee, D.B. Graves, and M.A. Lieberman, Plasma Sources Sci. Technol. 17, 015018 (2008)

[26] C.K. Birdsall and A.B. Langdon, Plasma Physics via Computer Simulation (IOP Publishing, London, 1991)

[27] D. Vender and R.W. Boswell, IEEE Trans. Plasma Sci. 18, 725 (1990)

[28] M. Surendra and D.B. Graves, IEEE Trans. Plasma Sci. 19, 144 (1991)

[29] P. Mertmann, D. Eremin, T. Mussenbrock, R.P. Brinkmann, and P. Awakowicz, Comp. Phys. Comm. 182, 2161 (2011)

[30] S. Rauf, J. Appl. Phys 87, 7647 (2000)

[31] A.C.F. Wu, M.A. Lieberman, and J.P. Verboncoeur, J. Appl. Phys 101, 056105 (2007)

[32] H.H. Goto, H.D. Löwe, and T. Ohmi, J. Vac. Sci. Technol. A 10, 3048, (1992)

[33] N. Nakano and T. Makabe, J. Phys. D: Appl. Phys. 28, 31, (1995)

[34] J.K. Lee, N.Y. Babaeva, H.C. Kim, O.V. Manuilenko, and J.W. Shon, IEEE Trans. on Plasma Sci. 32, 47, (2004)

[35] J.K. Lee, O.V. Manuilenko, N.Y. Babaeva, H.C. Kim, and J.W. Shon, Plasma Sources Sci. Technol., 14, 89, (2005)

[36] S. Rauf and M.J. Kushner, IEEE Trans. on Plasma Sci. 27, 1329 (1999)

[37] T. Mussenbrock, D. Ziegler, and R.P. Brinkmann, Phys. Plasmas 13, 083501 (2006)

[38] D. Ziegler, T. Mussenbrock, and R.P. Brinkmann, Phys. Plasmas 16, 023503 (2009)

[39] D. Ziegler, J. Trieschmann, T. Mussenbrock, R.P. Brinkmann, J. Schulze, U.Czarnetzki, E. Semmler, P. Awakowicz, D. O'Connell, and T. Gans, Plasma Sources Sci. Technol. 19, 045001 (2010)

[40] B.G. Heil, J. Schulze, T. Mussenbrock, R.P. Brinkmann, and U. Czarnetzki, IEEE Trans. on Plasma Sci. 36, 1404 (2008)

[41] B.G. Heil, U. Czarnetzki, R.P. Brinkmann, and T Mussenbrock, J. Phys. D: Appl. Phys. 41165202 (2008)

[42] Z. Donko, J. Schulze, B.G. Heil, U. Czarnetzki, J. Phys. D: Appl. Phys. 42, 025205 (2009)

[43] U. Czarnetzki, B.G. Heil, J. Schulze, Z. Donko, T. Mussenbrock, and R.P. Brinkmann, J. Phys.: Conf. Series 162, 012010 (2009)

[44] J. Schulze, E. Schngel, U. Czarnetzki, M. Gebhardt, R.P. Brinkmann, and T. Mussenbrock, Appl. Phys. Lett. 98, 031501 (2011)

[45] S.B. Wang and A.E. Wendt, J. Appl. Phys. 88, 643 (2000)

[46] S.B. Wang and A.E. Wendt, J. Vac. Sci. Technol. A 19, 2425 (2001)

[47] R. Silapunt, A. E. Wendt, K. Kirmse, and L. P. Losey, J. Vac. Sci. Technol. B 22, 826 (2004)

[48] F.L. Buzzi, Y.H. Ting, and A.E. Wendt, Plasma Sources Sci. Technol. 18, 025009 (2009)

[49] T. Baloniak, PhD Thesis, Ruhr University Bochum (2010)

[50] T. Baloniak, R. Reuter, C. Fltgen, and A. von Keudell J. Phys. D: Appl. Phys. 43, 055203 (2010)

[51] T. Baloniak, R. Reuter, and A. von Keudell, J. Phys. D: Appl. Phys. 43, 335201 (2010) 